\section{(2) \\ BRAZIULIAN JOURNAL \\ OF MEDICAL AND BIOLOGICAL RESEARCH}

www.bjournal.com.br
ISSN 0100-879X

Volume 45 (7) 565-680

July 2012

CLINICAL INVESTIGATION

Braz J Med Biol Res, July 2012, Volume 45(7) 578-582

doi: 10.1590/S0100-879X2012007500064

Pain pressure threshold algometry of the abdominal wall in healthy women

M.L.L.S. Montenegro, C.A. Braz, E.L. Mateus-Vasconcelos, J.C. Rosa-e-Silva, F.J. Candido-dos-Reis, A.A. Nogueira and O.B. Poli-Neto

The Brazilian Journal of Medical and Biological Research is partially financed by

\section{욛NPq}

da Ciência e Tecnologia

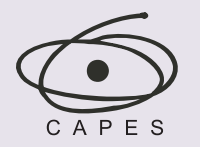

Ministério da Educação

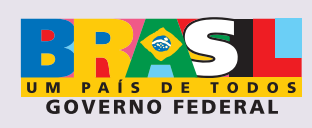

ATFAPESP

Institutional Sponsors

Ф SHIMADZU UNICAMP

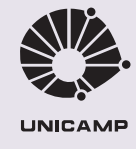

GOVERNO FEDERAL

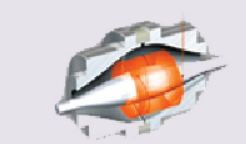

xplore High - Performance MS Orbitrap Technology In Proteomics \& Metabolomics analitica Thermo

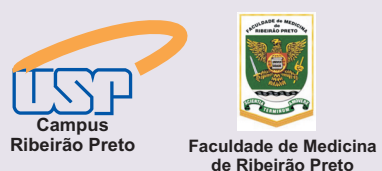

de Ribeirão Preto

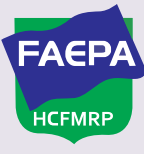




\title{
Pain pressure threshold algometry of the abdominal wall in healthy women
}

\author{
M.L.L.S. Montenegro2*, C.A. Braz², E.L. Mateus-Vasconcelos ${ }^{2 *}$, \\ J.C. Rosa-e-Silva ${ }^{2 *}$, F.J. Candido-dos-Reis ${ }^{2 *}$, A.A. Nogueira ${ }^{2 *}$ \\ and O.B. Poli-Neto ${ }^{1,2 *}$ \\ ${ }^{1}$ Departamento de Cirurgia e Anatomia, Faculdade de Medicina de Ribeirão Preto, \\ Universidade de São Paulo, Ribeirão Preto, SP, Brasil \\ 2Departamento de Ginecologia e Obstetrícia, Faculdade de Medicina de Ribeirão Preto, \\ Universidade de São Paulo, Ribeirão Preto, SP, Brasil
}

\begin{abstract}
The objective of this study was to determine the inter- and intra-examiner reliability of pain pressure threshold algometry at various points of the abdominal wall of healthy women. Twenty-one healthy women in menacme with a mean age of $28 \pm 5.4$ years (range: 19-39 years) were included. All volunteers had regular menstrual cycles (27-33 days) and were right-handed and, to the best of our knowledge, none were taking medications at the time of testing. Women with a diagnosis of depression, anxiety or other mood disturbances were excluded. Women with previous abdominal surgery, any pain condition or any evidence of inflammation, hypertension, smoking, alcoholism, or inflammatory disease were also excluded. Pain perception thresholds were assessed with a pressure algometer with digital traction and compression and a measuring capacity for $5 \mathrm{~kg}$. All points were localized by palpation and marked with a felt-tipped pen and each individual was evaluated over a period of 2 days in two consecutive sessions, each session consisting of a set of 14 point measurements repeated twice by two examiners in random sequence. There was no statistically significant difference in the mean pain threshold obtained by the two examiners on 2 diferent days (examiner $A: P=1.00$; examiner $B: P=0.75$; Wilcoxon matched pairs test). There was excellent/good agreement between examiners for all days and all points. Our results have established baseline values to which future researchers will be able to refer. They show that pressure algometry is a reliable measure for pain perception in the abdominal wall of healthy women.
\end{abstract}

Key words: Chronic pelvic pain; Pain pressure threshold; Myofascial pain syndrome; Reliability; Abdominal muscles

\section{Introduction}

Chronic pelvic pain (CPP) is a prevalent clinical condition among women (1-3), commonly described as continuous or intermittent pain in the anatomic pelvis (anterior abdominal wall at or below the umbilicus) that lasts for at least six months, is not exclusively related to menstruation or sexual intercourse, and is sufficiently severe to cause functional disability or to lead the patient to seek medical care (4). Although the more common diagnoses described in women with CPP are endometriosis, adhesions, irritable bowel syndrome, and painful bladder syndrome/interstitial cystitis, the involvement of the musculoskeletal system in the genesis and perpetuation of CPP has been increasingly demonstrated, particularly the abdominal myofascial pain syndrome (AMPS) characterized as intense and profound pain in the abdominal region originating from myofascial triggering points (MTrP) (5), which, in turn, are hyperirritable points usually localized within a skeletal muscle fascia or in the muscle covering fascia, and they can be classified as active or latent (6). AMPS usually affects women more than men (54 versus $45 \%$ ), and is more frequent among women aged $30-40$ years (7). The estimated prevalence is about $30 \%$ at primary medical care centers and $85-93 \%$ at centers specializing in the treatment of pain (6). However, there are few data in the literature regarding the real incidence and prevalence of myofascial pain syndrome specifically of abdominal origin. In our experience, about $15-30 \%$ of women with CPP attended by our group had AMPS. A possible explanation for this high prevalence may be the high

Correspondence: O.B. Poli-Neto, Departamento de Cirurgia e Anatomia, FMRP, USP, Av. Bandeirantes, 3900, 8ํandar, 14049-900 Ribeirão Preto, SP, Brasil. Fax: +55-16-3633-0946. E-mail: polineto@fmrp.usp.br

*These authors contributed equally to this study.

Received August 31, 2011. Accepted April 12, 2012. Available online April 27, 2012. Published July 2, 2012. 
rates of cesarean section surgery in Brazil (3).

The quantification of pain is important and pressure algometry has proven to be useful for identifying tender spots and trigger points in the head and neck and also for the assessment of the results of treatment (8). Fenton et al. (9) recently demonstrated that pain pressure threshold (PPT) testing can be a useful tool for objective quantification of local pain (9), and is also important as a method for monitoring women with CPP. However, few studies (10) have provided actual estimates of inter-and intra-examiner reliability in pressure algometry for the determination of pain thresholds at points on the abdominal wall of healthy women, particularly controlled according to the phase of the menstrual cycle. Therefore, the purpose of the present study was to determine the inter- and intra-examiner reliability of PPT algometry at various points of the abdominal wall of healthy women.

\section{Material and Methods}

The study was approved by the Research Ethics Committee of Hospital das Clínicas, Faculdade de Medicina de Ribeirão Preto, Universidade de São Paulo (HCFMRPUSP) and all subjects gave written informed consent to participate. Twenty-one healthy women with a mean age of $28 \pm 5.4$ years (range 19-39 years), mean weight of $56.2 \pm$ $6.3 \mathrm{~kg}$, mean height of $1.63 \pm 0.06 \mathrm{~m}$, and body mass index of $21.1 \pm 2.2 \mathrm{~kg} / \mathrm{m}^{2}$ were included in the study. All volunteers had regular menstrual cycles (27-33 days) and were right-handed and, to the best of our knowledge, none was taking medications (including hormonal contraceptives) at the time of testing. Women with a diagnosis of depression, anxiety or other mood disturbances were excluded. Women with previous abdominal surgery, any pain condition such as low back pain, migraine, or constipation, among others, or any evidence of inflammation, hypertension, smoking, alcoholism, or inflammatory disease were also excluded.

PPT, the minimal pressure (force) that induces pain, was assessed with an Instrutherm DD-500 pressure algometer, model DD200 with digital traction and compression and measuring capacity for $5 \mathrm{~kg}$ (Instrutherm Instrumentos de Medição Ltda., Brazil). The apparatus consists of a rubber disk 1 $\mathrm{cm}$ in diameter attached to the plunger of a pressure (force) gauge. The dial of the gauge is calibrated in $\mathrm{kg} / \mathrm{cm}^{2}$. To cover clinically significant structures in the abdomen, 14 points were specifically marked bilaterally (Figure 1). Additionally, we measured one point on the thenar region of the right hand as a control point. All points were localized by palpation and marked with a felt-tipped pen. Each individual was evaluated on 2 days in two consecutive sessions, each consisting of a set of 14-point measurements repeated three times. The average of the two closest values within each session was used for further calculations. The interval between the end of one session and the beginning of the next was approximately 20-30 min. The measurements were made at random with the test subject in the supine position. The subjects were assigned at random to one of four sequences carried out by examiner A (M.L.L.S.M.) and examiner B (E.L.M.V.; sequence 1: $B A A B$; sequence 2: BABA; sequence 3: $A B B A$; sequence $4: A B A B)$. All subjects were specifically instructed to indicate when the pressure became painful. The subjects were informed that the objective of the investigation was to determine the pain threshold, and not pressure or pain tolerance. A5-s rest was allowed between points tested. The application of the meter to $3 \mathrm{kgf}$ was initially demonstrated

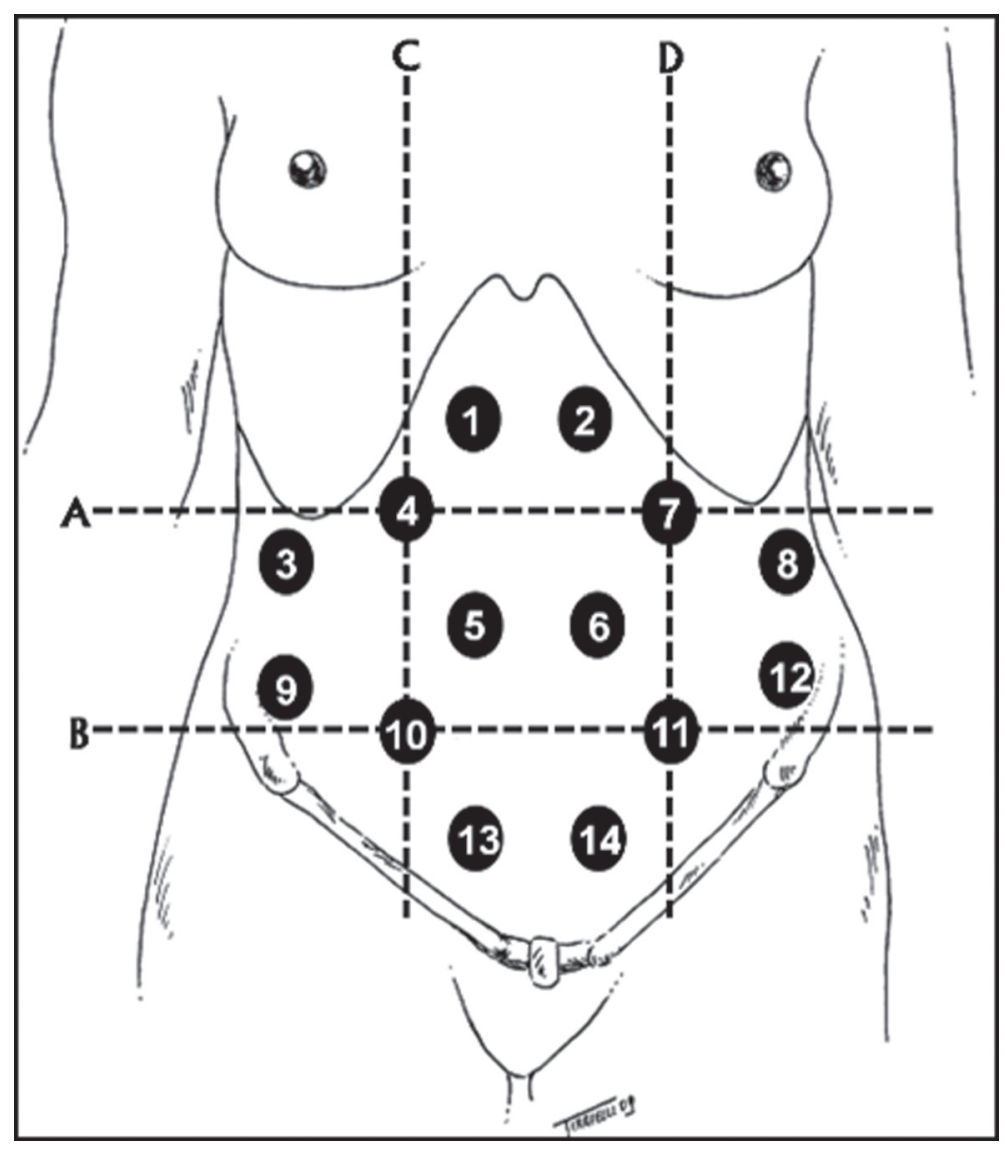

Figure 1. Location of the 14 points studied. Planes $A=$ subcostal; $B=$ transtubercular; $C=$ right hemiclavicular; $D=$ left hemiclavicular. Points were symmetrically distributed at correspondent areas. 
Table 1. Pain pressure threshold (PPT) measured by each examiner on 2 days.

\begin{tabular}{|c|c|c|c|c|c|c|c|c|}
\hline \multirow[t]{3}{*}{ Points } & \multicolumn{4}{|c|}{ PPT (day 1) } & \multicolumn{4}{|c|}{ PPT (day 2) } \\
\hline & \multicolumn{2}{|c|}{ Examiner $\mathrm{A}$} & \multicolumn{2}{|c|}{ Examiner B } & \multicolumn{2}{|c|}{ Examiner $\mathrm{A}$} & \multicolumn{2}{|c|}{ Examiner B } \\
\hline & Session 1 & Session 2 & Session 1 & Session 2 & Session 1 & Session 2 & Session 1 & Session 2 \\
\hline 1 & $2.81 \pm 1.94$ & $2.67 \pm 1.54$ & $2.49 \pm 1.43$ & $2.67 \pm 1.79$ & $2.41 \pm 1.55$ & $2.46 \pm 1.33$ & $2.47 \pm 1.26$ & 2.54 \\
\hline 2 & $2.51 \pm 1.63$ & $2.45 \pm 1.53$ & $2.58 \pm 1.57$ & $2.68 \pm 1.76$ & $2.26 \pm 1.58$ & $2.10 \pm 1.34$ & $2.20 \pm 1.33$ & $2.44 \pm 1.45$ \\
\hline 3 & $2.37 \pm 1.47$ & $2.62 \pm 1.45$ & $2.67 \pm 1.22$ & $2.86 \pm 1.14$ & $2.23 \pm 0.76$ & $2.58 \pm 1.14$ & $2.59 \pm 1.00$ & $2.93 \pm 1.14$ \\
\hline 4 & $2.54 \pm 1.47$ & $2.55 \pm 1.60$ & $2.67 \pm 1.17$ & $2.70 \pm 1.35$ & $2.39 \pm 0.99$ & $2.39 \pm 0.98$ & $2.46 \pm 1.04$ & $2.59 \pm 1.17$ \\
\hline 5 & $2.52 \pm 1.52$ & $2.52 \pm 1.57$ & $2.29 \pm 1.26$ & $2.38 \pm 1.31$ & $2.43 \pm 1.46$ & $2.41 \pm 1.53$ & $2.17 \pm 1.14$ & $2.25 \pm 1.16$ \\
\hline 6 & $2.21 \pm 1.53$ & $2.47 \pm 1.47$ & $2.36 \pm 1.27$ & $2.26 \pm 1.22$ & $2.30 \pm 1.42$ & $2.29 \pm 1.58$ & $2.02 \pm 0.92$ & $2.08 \pm 1.01$ \\
\hline 7 & $2.60 \pm 1.47$ & $2.76 \pm 1.58$ & $2.61 \pm 1.32$ & $2.69 \pm 1.30$ & $2.37 \pm 1.23$ & $2.50 \pm 1.16$ & $2.33 \pm 1.01$ & $2.54 \pm 1.07$ \\
\hline 8 & $2.46 \pm 1.40$ & $2.50 \pm 1.30$ & $2.51 \pm 1.33$ & $2.66 \pm 1.49$ & $2.26 \pm 1.04$ & $2.28 \pm 1.17$ & $2.43 \pm 1.06$ & $2.64 \pm 1.28$ \\
\hline 9 & $2.77 \pm 1.45$ & $2.79 \pm 1.53$ & $2.74 \pm 1.18$ & $2.94 \pm 1.53$ & $2.32 \pm 1.03$ & $2.54 \pm 1.25$ & $2.71 \pm 1.13$ & $2.89 \pm 1.18$ \\
\hline 10 & $2.72 \pm 1.62$ & $2.63 \pm 1.53$ & $2.50 \pm 1.40$ & $2.72 \pm 1.33$ & $2.39 \pm 0.95$ & $2.57 \pm 1.13$ & $2.44 \pm 1.03$ & $2.45 \pm 1.13$ \\
\hline 11 & $2.53 \pm 1.51$ & $2.74 \pm 1.59$ & $2.55 \pm 1.33$ & $2.62 \pm 1.54$ & $2.30 \pm 1.00$ & $2.56 \pm 1.37$ & $2.38 \pm 1.11$ & $2.57 \pm 1.24$ \\
\hline 12 & $2.69 \pm 1.50$ & $2.74 \pm 1.44$ & $2.75 \pm 1.31$ & $2.79 \pm 1.40$ & $2.55 \pm 1.14$ & $2.67 \pm 1.19$ & $2.71 \pm 1.15$ & $2.69 \pm 1.22$ \\
\hline 13 & $2.85 \pm 1.91$ & $2.74 \pm 1.85$ & $2.42 \pm 1.28$ & $2.71 \pm 1.84$ & $2.21 \pm 1.27$ & $2.41 \pm 1.44$ & $2.27 \pm 1.63$ & $2.35 \pm 1.59$ \\
\hline 14 & $2.77 \pm 2.05$ & $2.47 \pm 1.62$ & $2.59 \pm 1.61$ & $2.54 \pm 1.73$ & $2.45 \pm 1.55$ & $2.37 \pm 1.57$ & $2.25 \pm 1.13$ & $2.25 \pm 1.22$ \\
\hline
\end{tabular}

Data are reported as means \pm SD for 14 points measured on 21 women. The thenar region of the right hand was used as a control point. PPT is reported in $\mathrm{kg} / \mathrm{cm}^{2}$.

on the women's thenar eminence to reduce anxiety. The rate of pressure increase was approximately 1 $\mathrm{kg} \cdot \mathrm{cm}^{2} \cdot \mathrm{s}^{-1}$. All tests were conducted on women in the follicular phase of the menstrual cycle (4th to 7 th day) in an environment with controlled temperature $\left(24-26^{\circ} \mathrm{C}\right)$ between 7:00 and 9:00 am.

\section{Statistical analysis}

Data are reported as means $\pm S D$. Intraclass correlation coefficient (ICC), Bland Altman plots and SEM were used to measure reliability. The ICC is the fraction of variance that is caused by the variation between subjects. Thus, if the variance between tests (or examiners) is small compared to the variance between subjects, then ICC is close to 1. According to Fleiss (11), ICC values above 0.75 generally mean "excellent" reliability. The analyses were carried out using the SAS 9.0 software, with the level of significance set at $P<0.05$.

\section{Results}

The mean values obtained for each point on the 2 test days by the two examiners are presented in Table 1. There was no statistically significant difference in mean pain threshold values obtained by the two examiners on 2 days (examiner $A: P=1.00$; examiner $B$ : $P=0.75)$. There was excellent/good agreement between examiners regarding all days and points (Tables 2, 3, and 4). There was no evidence of temporal summation (the first
Table 2. Reliability of measures between regions according to examiner and session day.

\begin{tabular}{cccccc}
\hline \multirow{2}{*}{ Points } & \multicolumn{2}{c}{ ICC (day 1) } & & \multicolumn{2}{c}{ ICC (day 2) } \\
\cline { 2 - 3 } \cline { 5 - 6 } & Examiner A & Examiner B & & Examiner A & Examiner B \\
\hline Thenar & 0.91 & 0.75 & & 0.94 & 0.88 \\
1 & 0.89 & 0.91 & & 0.96 & 0.94 \\
2 & 0.85 & 0.95 & & 0.51 & 0.88 \\
3 & 0.93 & 0.89 & & 0.77 & 0.86 \\
4 & 0.91 & 0.93 & & 0.94 & 0.89 \\
5 & 0.94 & 0.98 & & 0.96 & 0.94 \\
6 & 0.96 & 0.92 & & 0.95 & 0.95 \\
7 & 0.94 & 0.96 & & 0.91 & 0.85 \\
8 & 0.89 & 0.95 & & 0.93 & 0.92 \\
9 & 0.94 & 0.84 & & 0.86 & 0.91 \\
10 & 0.96 & 0.91 & & 0.93 & 0.91 \\
11 & 0.95 & 0.93 & & 0.92 & 0.92 \\
12 & 0.96 & 0.98 & & 0.96 & 0.95 \\
13 & 0.96 & 0.87 & & 0.90 & 0.98 \\
14 & 0.93 & 0.95 & & 0.98 & 0.91 \\
\hline
\end{tabular}

ICC = intraclass correlation coefficient. The thenar region of the right hand was used as a control point.

and third measures were more frequently excluded), but we did not analyze this situation in particular because we did not consider it clinically important. 
Table 3. Reliability of measures between session days according to examiner and region.

\begin{tabular}{ccc}
\hline Points & \multicolumn{2}{c}{ ICC for 2 days } \\
\cline { 2 - 3 } & Examiner A & Examiner B \\
\hline Thenar & 0.79 & 0.70 \\
1 & 0.85 & 0.86 \\
2 & 0.57 & 0.86 \\
3 & 0.70 & 0.78 \\
4 & 0.78 & 0.72 \\
5 & 0.80 & 0.80 \\
6 & 0.89 & 0.80 \\
7 & 0.80 & 0.75 \\
8 & 0.83 & 0.73 \\
9 & 0.80 & 0.71 \\
10 & 0.77 & 0.68 \\
11 & 0.81 & 0.72 \\
12 & 0.79 & 0.78 \\
13 & 0.87 & 0.87 \\
14 & 0.90 & 0.85 \\
\hline
\end{tabular}

ICC = intraclass correlation coefficient. The thenar region of the right hand was used as a control point.

\section{Discussion}

Our results showed that the maximum PPT on the abdominal wall of healthy women was $2.93 \mathrm{~kg} / \mathrm{cm}^{2}$ and the minimum was $2.02 \mathrm{~kg} / \mathrm{cm}^{2}$. Thus, in women with CPP caused by AMPS the PPT should be lower. This is an important point because before the present study we did not know what the high and low thresholds of pain would be in women with CPP caused by AMPS. Thus, our results have also established baseline values to which future researchers will be able to refer.

In the present study, both inter-and intra-examiner agreement was also found to be good, except for point two, maybe because of its difficult location and its proximity to cartilage and bone structures. Anyway, the measures in the lower quadrants of the abdomen were reliable. This agreement is important because clinically the PPT gauge is used as a semi-quantitative method for the measurement of intensity of tenderness and for the location of tender spots, to prove to the patient that tenderness, pain, MTrP and their sensitivity correlate during long-term follow-up of change in pain, and evaluation of activity in inflamed joints. In addition, it is used for medicolegal documentation since the reproducibility of pressure threshold indicates that the records of pain intensity are quantitative and objective $(10,12)$. Nevertheless, the pressure algometer cannot be used to diagnose AMPS since it cannot accurately distinguish the generation of pain, although it can help monitor the progress of treatment in an
Table 4. Reliability of measures between examiners according to session day and region.

\begin{tabular}{ccc}
\hline Points & \multicolumn{2}{c}{ ICC for two examiners } \\
\cline { 2 - 3 } & Day 1 & Day 2 \\
\hline Thenar & 0.79 & 0.90 \\
1 & 0.89 & 0.91 \\
2 & 0.88 & 0.68 \\
3 & 0.88 & 0.82 \\
4 & 0.88 & 0.89 \\
5 & 0.92 & 0.89 \\
6 & 0.90 & 0.85 \\
7 & 0.90 & 0.81 \\
8 & 0.85 & 0.86 \\
9 & 0.86 & 0.85 \\
10 & 0.91 & 0.86 \\
11 & 0.93 & 0.88 \\
12 & 0.92 & 0.91 \\
13 & 0.90 & 0.91 \\
14 & 0.93 & 0.88 \\
\hline
\end{tabular}

ICC = intraclass correlation coefficient. The thenar region of the right hand was used as a control point.

objective manner. This is an important factor mainly when it is necessary to measure pain objectively, as done in scientific research. Also this is an inexpensive, easy and reproducible method for the measurement of pain threshold.

We did not observe any evidence of reduced PPT with repeated testing in the same point. However, we opted to exclude the more discrepant measure in order to ensure the methodological rigor of the study design. Curiously, the measurements excluded from the analysis were, more often, the first or the third ones, which were generally slightly lower than the others. We may attribute this to the women's adaption to the test and not to temporal summation of pain perception, although this question was not analyzed in detail. Xu et al. (13) had observed that MTrPs are one of the important peripheral pain generators and initiators for central sensitization. However, they did not observe the same results for non-trigger points in healthy subjects (13). These points, when stimulated by needle electromyography for $8 \mathrm{~min}$, do not induce any evidence of central sensitization. Thus, we do not believe that the measurement of PPT alone would be enough to induce sensitization.

\section{Acknowledgments}

We wish to thank Prof. Luís Fernando Tirapelli, Department of Surgery and Anatomy, Faculty of Medicine of Ribeirão Preto, University of São Paulo, for the illustration. 


\section{References}

1. Zondervan KT, Yudkin PL, Vessey MP, Dawes MG, Barlow $\mathrm{DH}$, Kennedy SH. Prevalence and incidence of chronic pelvic pain in primary care: evidence from a national general practice database. Br J Obstet Gynaecol 1999; 106: 11491155.

2. Zondervan KT, Yudkin PL, Vessey MP, Jenkinson CP, Dawes $\mathrm{MG}$, Barlow DH, et al. The community prevalence of chronic pelvic pain in women and associated illness behaviour. $\mathrm{Br} J$ Gen Pract 2001; 51: 541-547.

3. Silva GPOG, Nascimento AL, Michelazzo D, Alves-Junior FF, Rocha MG, Rosa-e-Silva JC, et al. High prevalence of chronic pelvic pain in women in Ribeirão Preto, Brazil and direct association with abdominal surgery. Clinics 2011; 66: 1307-1312.

4. ACOG Practice Bulletin No. 51. Chronic pelvic pain. Obstet Gynecol 2004; 103: 589-605.

5. Simons DG, Travell JG, Simons LS, Cummings BD. Myofascial pain and dysfunction: the trigger point manual. 2nd edn. London: Williams and Wilkins; 1999.

6. Sharp HT. Myofascial pain syndrome of the abdominal wall for the busy clinician. Clin Obstet Gynecol 2003; 46: 783-788.

7. Tough EA, White AR, Richards S, Campbell J. Variability of criteria used to diagnose myofascial trigger point pain syndrome - evidence from a review of the literature. Clin $J$ Pain 2007; 23: 278-286.

8. Antonaci F, Sand T, Lucas GA. Pressure algometry in healthy subjects: inter-examiner variability. Scand J Rehabil Med 1998; 30: 3-8.

9. Fenton BW, Palmieri PA, Durner C, Fanning J. Quantification of abdominal wall pain using pain pressure threshold algometry in patients with chronic pelvic pain. Clin J Pain 2009; 25: 500-505.

10. Fischer AA. Pressure algometry over normal muscles. Standard values, validity and reproducibility of pressure threshold. Pain 1987; 30: 115-126.

11. Fleiss JL. The design and analysis of clinical experiments. Hoboken: John Wiley \& Sons; 1999.

12. Fischer AA. Pressure threshold meter: its use for quantification of tender spots. Arch Phys Med Rehabil 1986; 67: 836-838.

13. $\mathrm{Xu} Y M$, Ge HY, Arendt-Nielsen L. Sustained nociceptive mechanical stimulation of latent myofascial trigger point induces central sensitization in healthy subjects. J Pain 2010; 11: 1348-1355. 